\title{
Post-weaning high-fat diet accelerates kidney injury, but not hypertension programmed by maternal diabetes
}

\author{
Yessoufou Aliou', Min-Chun Liao', Xin-Ping Zhao', Shiao-Ying Chang', Isabelle Chenier', Julie R. Ingelfinger ${ }^{2}$ and \\ Shao-Ling Zhang ${ }^{1}$
}

BACKGROUND: The aim of this study was to establish the underlying mechanisms by which a post-weaning high-fat diet (HFD) accelerates the perinatal programming of kidney injury occurring in the offspring of diabetic mothers.

METHODS: Male mice, offspring of nondiabetic and diabetic dams were fed with normal diet (ND) or HFD from 4 to 20 wk of age. Rat renal proximal tubular cells were used in vitro.

RESULTS: On ND, the offspring of dams with severe maternal diabetes had an intrauterine growth restriction (IUGR) phenotype and developed mild hypertension and evidence of kidney injury in adulthood. Exposing the IUGR offspring to HFD resulted in rapid weight gain, catch-up growth, and later to profound kidney injury with activation of renal TGF $\beta 1$ and collagen type IV expression, increased oxidative stress, and enhanced renal lipid deposition, but not systemic hypertension. Given our data, we speculate that HFD or free fatty acids may accelerate the process of perinatal programming of kidney injury, via increased CD36 and fatty acid-binding protein 4 expression, which may target reactive oxygen species, nuclear factor-kappa B, and TGF $\beta 1$ signaling in vivo and in vitro.

CONCLUSION: Early postnatal exposure to overnutrition with a HFD increases the risk of development of kidney injury, but not hypertension, in IUGR offspring of dams with maternal diabetes.

D iabetes during pregnancy, whether gestational or pregestational diabetes (type 1 or type 2), results in offspring at high risk of developing hypertension, cardiovascular disease, and chronic kidney disease in adult life. This phenomenon, termed perinatal programming, in which intrauterine events are associated with later adverse changes, has attracted much attention (1-3). Substantial epidemiologic data have also suggested that the offspring whose mothers were diabetic during pregnancy are susceptible to metabolic disturbances induced by postnatal overnutrition, as seen with high-fat diet (HFD) or with increased caloric intake in early life (1-4).

Women who have diabetes during pregnancy and/or are obese and hyperinsulinemic are at risk of delivering macrosomic newborns (high birth weight), and both shortand long-term outcomes of macrosomic neonates are influenced by postnatal overnutrition (1-4). In high-birth-weight neonates, subsequent growth in infancy and risk of becoming obese or diabetic are directly and linearly linked-e.g., the higher the birth weight, the greater the risk of overweight and metabolic disturbances later in life (1-4). In contrast, pregnant women with severe, uncontrolled diabetes or diabetic complications, such as diabetic nephropathy and/or retinopathy, are at high risk of having a microsomic fetus (i.e., a fetus with intrauterine growth restriction (IUGR)) $(5,6)$. Such infants may be markedly small for dates; but many develop excessive weight gain and increased fat deposition in early infancy, a proxy for neonatal overnutrition $(7,8)$. However, the long-term outcome of IUGR offspring who experience overnutrition in early life is incompletely delineated.

Previously, we demonstrated in a mouse model that severe maternal diabetes (defined as maternal blood glucose concentration $\sim 30 \mathrm{mmol} / \mathrm{l}$ ) is linked to IUGR in offspring (mean decrease $20 \%$ of birth weight). Such offspring showed impaired nephrogenesis resulting in nascent nephron deficiency in neonate and manifested hypertension, glucose intolerance, and kidney injury in adulthood. The possible mechanisms involved in those phenomena include reactive oxygen species (ROS) elevation and activation of the nuclear factor-kappa B (NF$\mathrm{kB})$, TGF $\beta 1$, and $\mathrm{p} 53$ pathways (9-13).

Compelling evidence from both experimental (14-16) and human studies $(17,18)$ have suggested that perirenal and/or visceral fat depots may mediate the development of chronic kidney disease and hypertension. CD36 (19-22) and fatty acid-binding protein 4 (Fabp4) (23-27) may mediate chronic inflammation, insulin resistance, oxidant stress, and fibrosis involved in proatherogenic hyperlipidemic states such as obesity. In this study, we followed these affected IUGR offspring from 4 to $20 \mathrm{wk}$ of age and compared the metabolic impacts of post-weaning diet (HFD vs. normal diet (ND)) on handling of lipoproteins, hypertension, and renal function. Focusing on the kidney, we hypothesized that feeding the affected IUGR offspring with HFD after weaning would exacerbate

\footnotetext{
The first two authors contributed equally to this work.

'Centre de recherche du Centre hospitalier de I'Universite de Montreal (CRCHUM), Universite de Montreal, Montréal, Quebec, Canada; ${ }^{2}$ Pediatric Nephrology Unit, Massachusetts General Hospital and Harvard Medical School Boston, Boston, Massachusetts. Correspondence: Shao-Ling Zhang (shao.ling.zhang@umontreal.ca) Received 10 January 2015; accepted 24 August 2015; advance online publication 9 December 2015. doi:10.1038/pr.2015.236
} 
the metabolic perturbations and kidney injury in adulthood; we also hypothesized that alteration of renal CD36 and Fabp4 gene expression is associated with these adverse effects.

\section{RESULTS}

\section{Growth Curves of Offspring}

We compared the growth pattern of four subgroups of male offspring of nondiabetic (control) and diabetic dams-e.g., control offspring on ND (Con-ND, $N=17$ ); control offspring on HFD (Con-HF, $N=15$ ); diabetic offspring on ND (Dia-ND, $N=12$ ), and diabetic offspring on HFD (Dia-HF, $N=12)$. Similar to previous reports $(9-11)$, Dia-ND mice born with IUGR continued to have lower body weight (BW, g) throughout life (mean BW in cross-section time-point measurement decreased 20\%; $P \leq 0.01$; Figure 1a). HFD increased BW of both Con-HF and Dia-HF animals over time. Offspring of diabetic dams were more sensitive to HFD as compared to those of control dams, as they showed the steepest climb in BW-i.e., a catch-up pattern from 4 to $5 \mathrm{wk}$ $(P<0.05$; Figure 1c). In contrast, significant $\mathrm{BW}$ gain in
Con-HF mice (vs. Con-ND mice) began only at $8 \mathrm{wk}$ of age $(P<0.05$; Figure 1b).

\section{Metabolic Parameters}

At $20 \mathrm{wk}$, as compared with Con-ND mice, Dia-ND animals had increased plasma levels of nonesterified fatty acids (NEFA) (Figure 1d; $P<0.01$ ), triglycerides (Figure 1e; $P<0.01$ ), and cholesterol (Figure 1f; $P<0.05$ ), but not insulin (Figure 1g). HFD was associated with elevated plasma levels of NEFA $(P<0.05)$, triglycerides $(P<0.05)$, cholesterol $(P<0.01)$, and insulin $(P<0.01)$ in Con-HF animals, but this was not seen in Dia-HF groups (Figure $1 \mathbf{d}-\mathrm{g}$ ). Intraperitoneal glucose tolerance test (Figure $\mathbf{1 h}, \mathbf{i}$ ) and insulin sensitivity test (IST) (Figure 1j) were performed at $19 \mathrm{wk}$ before animals were killed. The fasting blood glucose concentration $(\mathrm{mmol} / \mathrm{l})$ at baseline of the four subgroups did not differ from each other (Con-ND $(8.31 \pm 0.34)$ vs. Dia-ND $(8.0 \pm 0.36)$; Con-HF $(10.45 \pm 0.47)$ vs. Dia-HF (9.71 \pm 0.53$)$; Figure $1 \mathbf{h})$. HFD impaired glucose tolerance in both Con-HF and Dia-HF offspring at similar levels (Figure 1h,i; $P<0.01$ ), despite the fact that Dia-ND already a

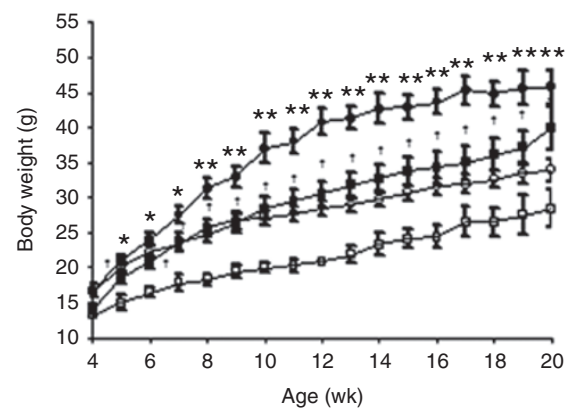

b

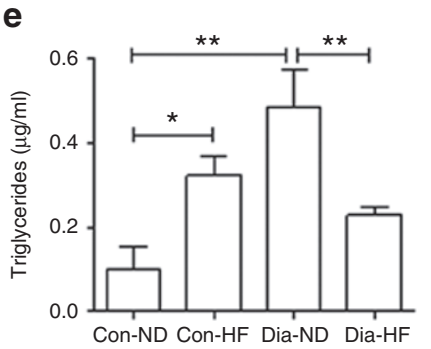

C d

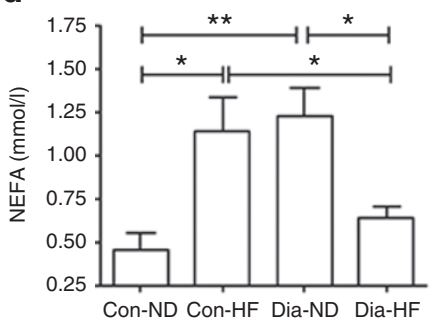

h

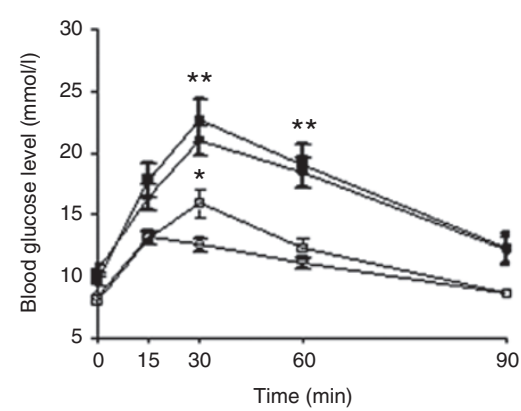

i

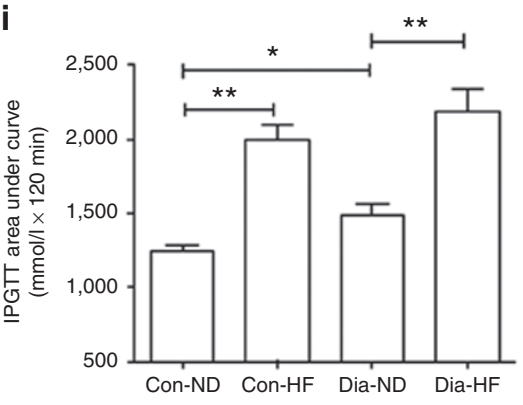

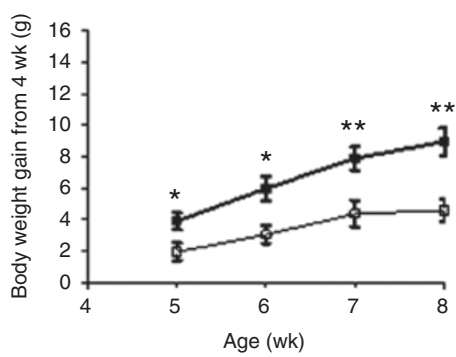

g

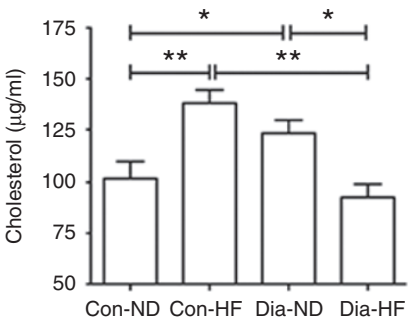

j

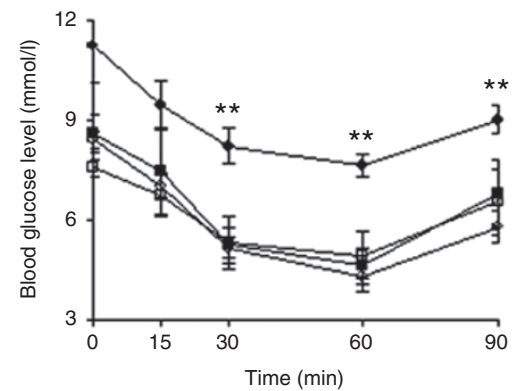

Figure 1. Metabolic parameters in offspring. (a) Growth follow-up in four subgroups of male offspring from the age of 4 to 20 wk. (O) Con-ND; (O) Con-HF; ( $\square$ ) Dia-ND; $(\square)$ Dia-HF; ${ }^{*}, P \leq 0.05 ;{ }^{* *} P \leq 0.01$ vs. Con-ND; ${ }^{+} P \leq 0.01$ vs. Dia-ND. (b,c) Body weight gain in male offspring from the age of 4 to 8 wk (b:

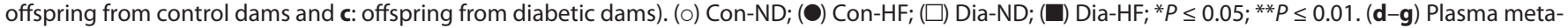
bolic profile measurements (d: NEFA (mmol/l); e: triglycerides $(\mathrm{mg} / \mathrm{ml})$; f: cholesterol $(\mu \mathrm{g} / \mathrm{ml})$; and $\mathbf{g}$ : insulin $(\mathrm{ng} / \mathrm{ml})$ ) in four subgroups of 20 -wk-old male offspring. ${ }^{*} P \leq 0.05$; ${ }^{* *} P \leq 0.01$. (h) IPGTT measurement. (o) Con-ND; (O) Con-HF; ( $\square$ ) Dia-ND; ( $\square$ ) Dia-HF; * $P \leq 0.05$; ** $\leq 0.01$ vs. Con-ND. (i) IPGTT area

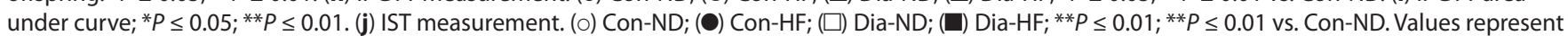
the mean \pm SEM. IPGTT, intraperitoneal glucose tolerance test. 


\section{Articles | Aliou et al.}

displayed a mild degree of impaired glucose tolerance $(P<0.05)$ when compared with Con-ND, as we also showed previously (10). Con-HF animals showed less insulin sensitivity through the entire IST procedure $(P<0.01)$, while a similar IST pattern was observed for the other three groups (Figure 1j).

\section{Mean Systolic Blood Pressure}

Longitudinal measurement of systolic blood pressure (SBP) (Figure 2a), recorded from 6 to $20 \mathrm{wk}$ of age, revealed that Dia-ND have significantly higher SBP over the follow-up period when compared with Con-ND, as previously reported (10). HFD animals displayed normal SBP ( $\mathrm{mm} \mathrm{Hg}$ ) at 10 to $20 \mathrm{wk}$ of age, irrespective of whether they were offspring of dams with maternal diabetes or not (Figure 2a).

\section{Kidney Function and Adipocyte Morphology}

As compared with Con-ND mice, Dia-ND animals exhibited significantly increased $(P \leq 0.05)$ urinary albumin/creatinine ratio (Figure $2 \mathbf{b}$ ) and glomerular filtration rate (GFR; $P \leq 0.01$; Figure 2c).

HFD was associated with a significantly increased albu$\mathrm{min} /$ creatinine ratio $(P \leq 0.05)$ in both groups of offspring compared with respective ND groups (Figure $2 \mathbf{b}$ ). However, HFD was not associated with changes of GFR/ tibia length (TL) ratio in either control or diabetic animals (Figure 2c). The kidney weight/TL $(\mathrm{mm})$ ratio among the four groups of animals remains unchanged either in ND or HFD condition.

As compared with Con-ND, Dia-ND mice had a decreased number of adipocytes $(P<0.01)$ collected from both perirenal and visceral fat. These changes were more pronounced in HFD offspring (Con-HF, $P<0.01$; Dia-HF, $P<0.05$; Figure 2d,e).

\section{Renal Expression of Extracellular Matrix Protein}

Consistent with previous reports $(9,10)$, Dia-ND offspring at 20 wk showed enhanced extracellular matrix protein accumulation seen by periodic acid-Schiff (Figure 3a) and Masson's trichrome (Figure $3 \mathbf{b}$ ) staining, resulting in glomerulosclerosis $(P<0.01$; Figure $3 \mathrm{~h})$ and tubulointerstitial injury $(P<0.05$; Figure 3i).

Our immunohistochemistry (IHC) data indicate that the kidneys of Dia-ND offspring also had increased expression of TGF $\beta 1$ (Figure 3c) and collagen type IV (Figure 3d) localized to both glomeruli $(P<0.01$; Figure $3 \mathbf{j})$ and tubulointerstitium $(P<0.05$; Figure 3k). And most importantly, HFD enhanced a

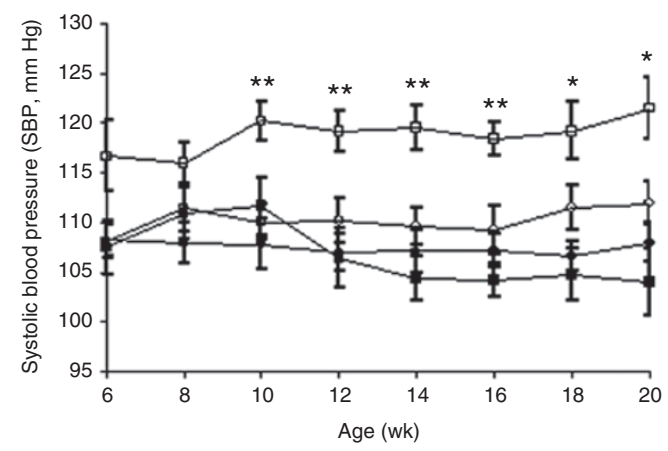

b

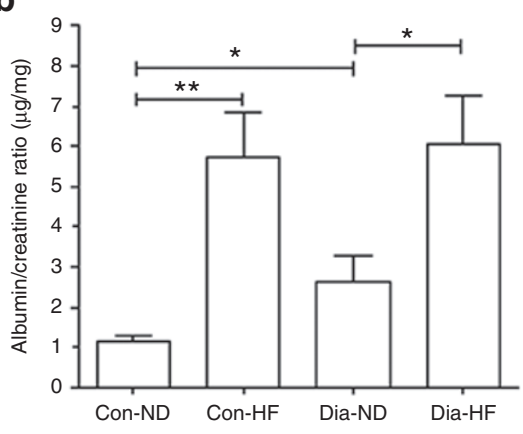

C

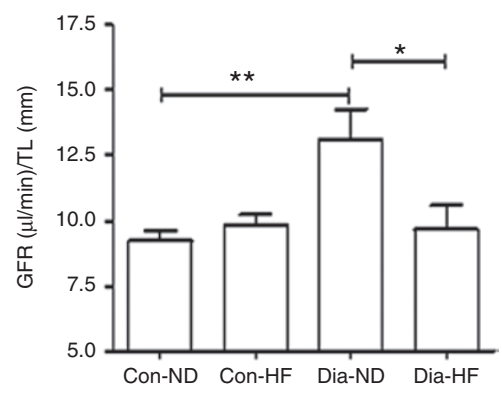

d

Con-ND

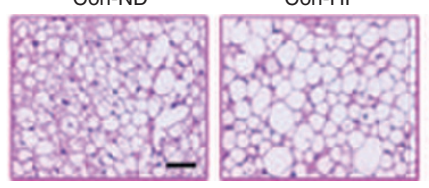

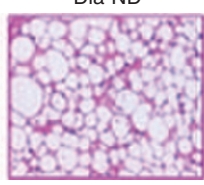

Dia-HF
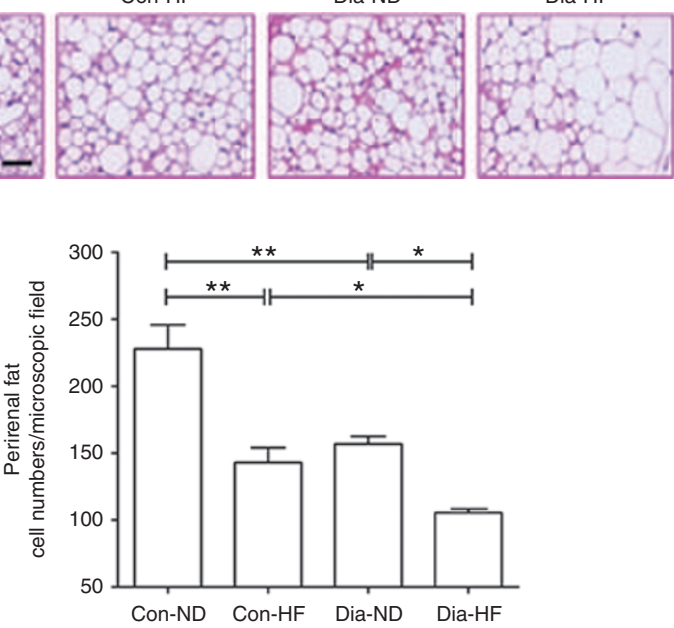

e

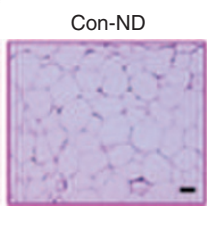

Con-HF
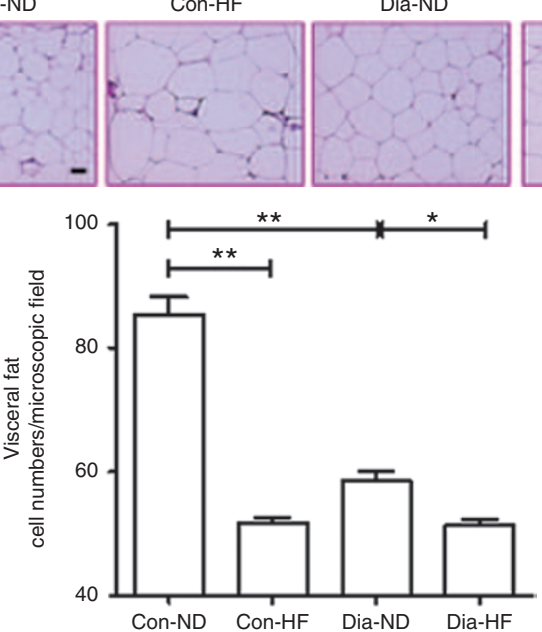

Dia-HF

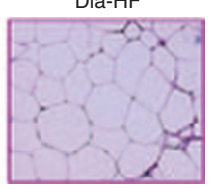

Figure 2. Blood pressure, renal function, and adipocyte morphology. (a) Longitudinal SBP measurement. (o) Con-ND; (O) Con-HF; ( $\square$ ) Dia-ND; $(\square)$ Dia-HF; ${ }^{*} P \leq 0.05 ;{ }^{* *} P \leq 0.01$ vs. Con-ND. (b) Urinary ACR measurement; ${ }^{*} P \leq 0.05 ;{ }^{* *} P \leq 0.01$. (c) GFR/TL ratio; ${ }^{*} P \leq 0.05 ;{ }^{*} P \leq 0.01$. (d) H\&E staining- perirenal fat (magnification: $\times 200$ ) with semi-quantitation of adipocyte number per microscopic field $\left(N=10\right.$ fields per animal). ${ }^{*} P \leq 0.05$; ${ }^{* *} P \leq 0.01$. (e) H\&E staining- visceral fat (magnification: $\times 100$ ) with semi-quantitation of adipocyte number per microscopic field $\left(N=10\right.$ fields per animal). ${ }^{*} P \leq 0.05 ; * * \leq 0.01$. Values represent the mean \pm SEM. ACR, albumin/creatinine ratio; GFR, glomerular filtration rate; H\&E, hematoxylin and eosin; SBP, systolic blood pressure; TL, tibia length. 

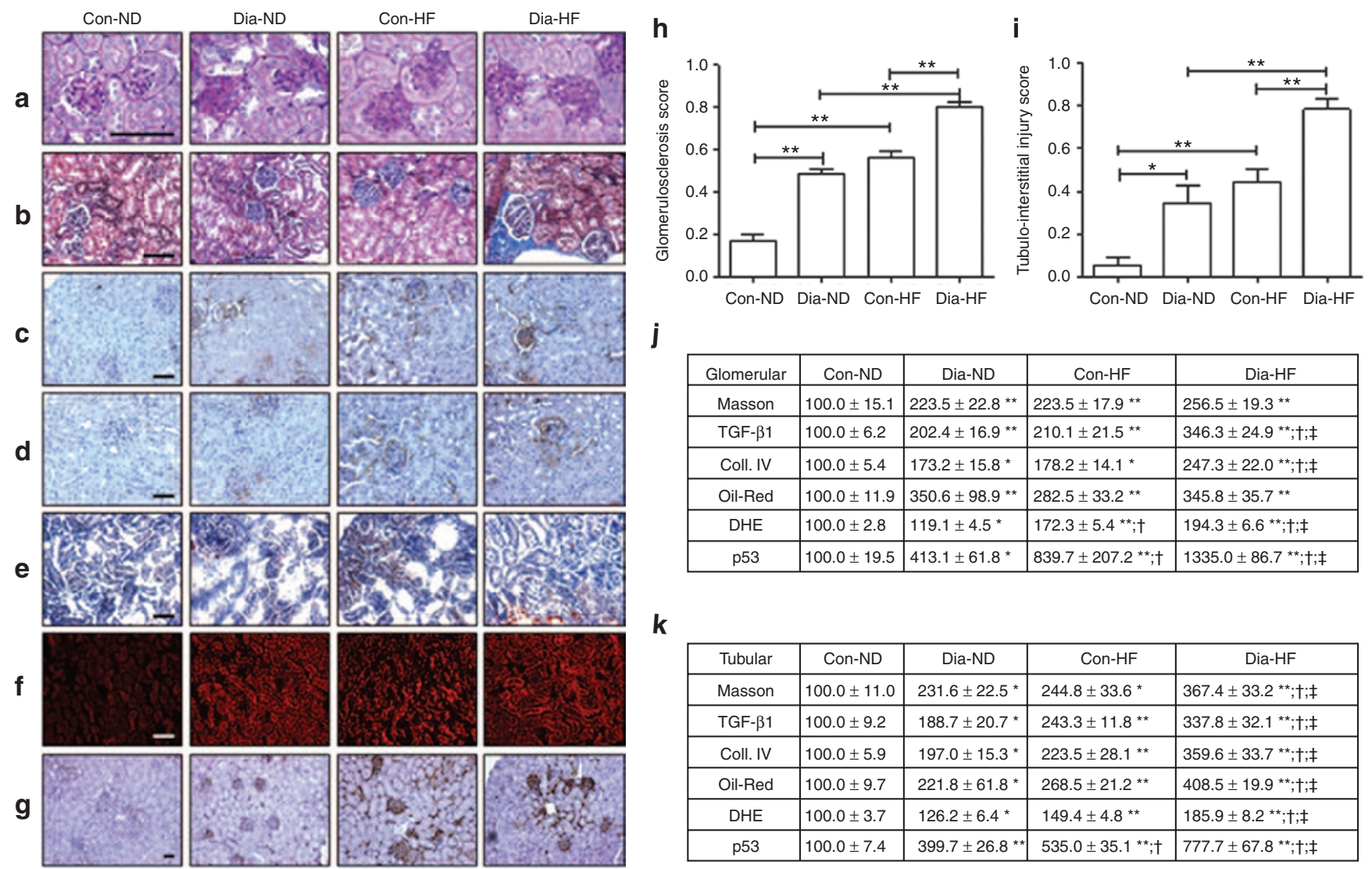

j

\begin{tabular}{|c|c|c|c|c|}
\hline Glomerular & Con-ND & Dia-ND & Con-HF & Dia-HF \\
\hline Masson & $100.0 \pm 15.1$ & $223.5 \pm 22.8$ ** & $223.5 \pm 17.9^{* *}$ & $256.5 \pm 19.3^{* *}$ \\
\hline TGF- $\beta 1$ & $100.0 \pm 6.2$ & $202.4 \pm 16.9$ ** & $210.1 \pm 21.5$ ** & $346.3 \pm 24.9^{* *} ; \dagger ; \ddagger$ \\
\hline Coll. IV & $100.0 \pm 5.4$ & $173.2 \pm 15.8$ * & $178.2 \pm 14.1$ * & $247.3 \pm 22.0^{* *} ; \dagger ; \ddagger$ \\
\hline Oil-Red & $100.0 \pm 11.9$ & $350.6 \pm 98.9$ ** & $282.5 \pm 33.2^{* \star}$ & $345.8 \pm 35.7^{\star \star}$ \\
\hline DHE & $100.0 \pm 2.8$ & $119.1 \pm 4.5^{*}$ & $172.3 \pm 5.4^{* *} ; \dagger$ & $194.3 \pm 6.6^{* *} ; \dagger ; \ddagger$ \\
\hline $\mathrm{p} 53$ & $100.0 \pm 19.5$ & $413.1 \pm 61.8$ * & $839.7 \pm 207.2^{* *} ; \dagger$ & $1335.0 \pm 86.7^{* *} ; \dagger ; \ddagger$ \\
\hline
\end{tabular}

k

\begin{tabular}{|c|c|c|c|c|}
\hline Tubular & Con-ND & Dia-ND & Con-HF & Dia-HF \\
\hline Masson & $100.0 \pm 11.0$ & $231.6 \pm 22.5$ * & $244.8 \pm 33.6$ * & $367.4 \pm 33.2^{* * ;} ; ; \neq \neq$ \\
\hline TGF- $\beta 1$ & $100.0 \pm 9.2$ & $188.7 \pm 20.7^{*}$ & $243.3 \pm 11.8$ ** & $337.8 \pm 32.1^{\star *} ; \dagger ; \ddagger$ \\
\hline Coll. IV & $100.0 \pm 5.9$ & $197.0 \pm 15.3$ * & $223.5 \pm 28.1$ ** & $359.6 \pm 33.7^{* *} ; \dagger ; \ddagger$ \\
\hline Oil-Red & $100.0 \pm 9.7$ & $221.8 \pm 61.8$ * & $268.5 \pm 21.2^{* *}$ & $408.5 \pm 19.9 * * ; ; ; \ddagger$ \\
\hline DHE & $100.0 \pm 3.7$ & $126.2 \pm 6.4^{*}$ & $149.4 \pm 4.8^{\text {** }}$ & $185.9 \pm 8.2^{* *} ; \dagger ; \ddagger$ \\
\hline $\mathrm{p} 53$ & $100.0 \pm 7.4$ & $399.7 \pm 26.8$ ** & $535.0 \pm 35.1^{* \star} ; \dagger$ & $777.7 \pm 67.8^{* *} ; \dagger ; \ddagger$ \\
\hline
\end{tabular}

Figure 3. Renal morphology. (a) PAS staining (magnification: $\times 600$ ). (b) Masson's trichrome staining (magnification: $\times 200)$. (c) IHC-TGF $\beta 1$ staining (magnification: $\times 200$ ). (d) IHC-collagen type IV (magnification: $\times 200$ ). (e) Oil red deposition (magnification: $\times 200)$. (f) DHE staining (magnification: $\times 200)$. (g) IHC-p53 staining (magnification: $\times 100$ ). (h) Classic scoring of glomerulosclerosis based on PAS images. Grade 0, normal glomeruli; grade 1, presence of mesangial expansion/thickening of the basement membrane. (i) Classic scoring of tubulointerstitial injury based on PAS images. Grade 0, normal tubules; grade 1, the tubulointerstitial lesions involving less than $25 \%$ of the field. (j-k) Semi-quantitation of the relative staining values in both (j) glomerular and (k) tubular compartment. The value in Con-ND animals was $100 \% \pm$ SEM. ${ }^{*} P \leq 0.05 ;{ }^{* *} P \leq 0.01$ vs. Con-ND; ${ }^{\dagger} P \leq 0.05$ vs. Dia-ND; ${ }^{\ddagger} P \leq 0.05$ vs. Con-HF. Values represent the mean $\pm S E M$. DHE, dihydroethidium; PAS, periodic acid-Schiff.

these changes in both groups of offspring $(P<0.01)$, particularly in the kidneys of Dia-HF mice.

\section{Oil Red Staining, Oxidative Stress, and Apoptosis in Kidney}

Within the kidneys of HFD animals (Con-HF vs. Dia-HF) at $20 \mathrm{wk}$ of age, there was increased oil red staining (Figure 3e), elevated oxidative stress on dihydroethidium staining (e.g., the nonfluorescent dihydroethidium is oxidized to fluorescent ethidium by superoxide anion $\left(\mathrm{O}_{2}^{--}\right)$) (Figure $3 \mathrm{f}$ ) and augmented apoptotic events on p53-IHC staining (Figure 3g) in both glomeruli $(P<0.05$; Figure $3 \mathbf{j})$ and tubulointerstitium $(P<0.05$; Figure $3 \mathbf{k})$.

\section{Renal CD36 and Fabp4 Expression}

As compared with Con-ND mice, renal CD36 (Figure 4a, mRNA; Figure 4c, protein) and Fabp4 (Figure 4b, mRNA; Figure 4d, protein) expression were increased in renal cortex in Dia-ND $(P<0.05)$ and both HFD-fed groups (Con-HF vs. Dia-HF). Moreover, IHC staining (Figure 5) revealed that increased CD36 and Fabp4 were predominantly localized in the proximal tubular cells, particularly in the kidneys of Dia-HF mice (Figure 5a) colocalizing with aquaporin 1 immunostaining (Figure 5b,c).

\section{In Vitro Studies}

We performed in vitro experiments using bovine serum albumin (BSA)-bound sodium palmitate (BSA-PA) to treat immortalized rat renal proximal tubular cells (IRPTCs) (9) to confirm our in vivo observations. BSA-PA stimulated both CD36 and Fapb4 protein expression in a dose-dependent manner (Figure 6a) in IRPTCs. Moreover, BSA-PA (125 $\mu \mathrm{mol} / \mathrm{l})$ was associated with significantly induced ROS generation (Figure $6 \mathbf{b} ; P<0.01$ ), increased apoptosis (Caspase 3 activity, Figure $6 c, P<0.01$; Hoechst staining, Figure $6 \mathrm{~d}, P<0.01$; and phosphorylation of p53, Figure 6e) and augmented NF-kB (i.e., p50/p65) as well as TGF $\beta 1$ protein expression (Figure 6e, $P<0.01)$ in IRPTCs.

\section{DISCUSSION}

This study indicates that IUGR offspring of diabetic dams postweaning fed with HFD showed "rapid" weight gain, catch-up growth, and subsequently displayed features of profound kidney injury associated with increased CD36 and Fabp4 expression. However, they did not have hypertension in adulthood.

In agreement with most human and experimental observations $(1-3,7,8)$, we observed "rapid" catch-up growth in young 

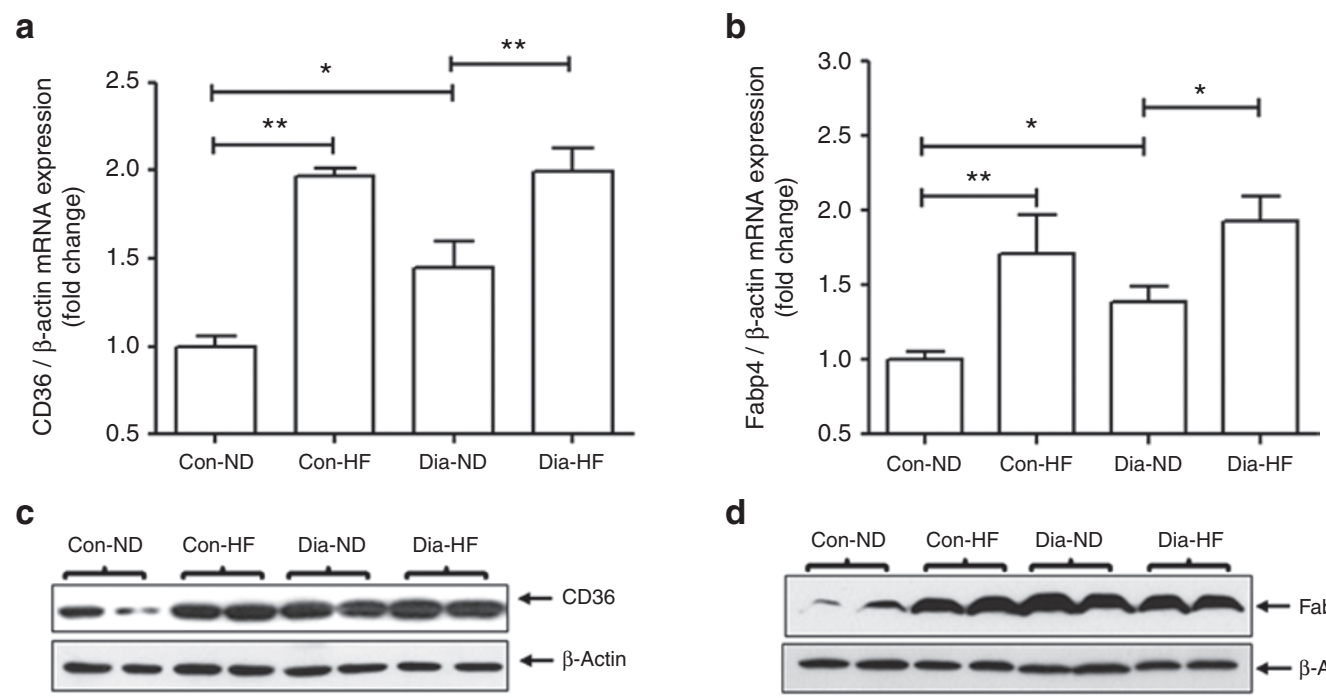

d
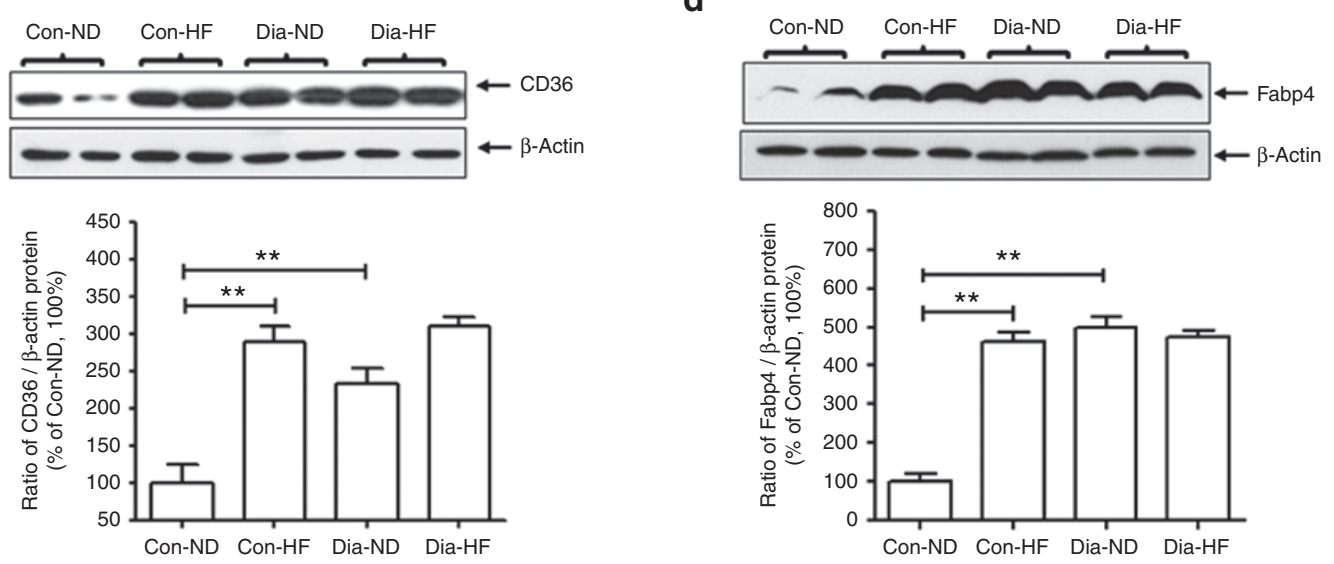

Figure 4. Renal CD36 \& Fabp 4 gene expression. (a-c) Renal CD36 and (b-d) Fabp4 gene expression in the renal cortex of four subgroups of 20-wk-old male offspring. ( $(\mathbf{a}$ and $\mathbf{b})$ qRT-PCR analysis. (c and $\mathbf{d}$ ) Western blot analysis. The relative densities of CD36 and Fabp4 were compared with their own $B$-actin mRNA or protein. The values of Con-ND animals were considered as $100 \%$. Each point represents the mean \pm SEM of three independent experiments. ${ }^{*} P \leq 0.05 ;{ }^{* *} P \leq 0.01$.

Dia-HF offspring from 5 to 8 wk of age. Con-HF offspring became obese over time, with elevated plasma lipids at $20 \mathrm{wk}$ of age (i.e., NEFA, triglycerides, and cholesterol), whereas Dia-HF offspring had similar BW and lipid levels as Con-ND at $20 \mathrm{wk}$ of age, underscoring the finding that postnatal HFD feeding has a differential impact on the growth pattern in the offspring of both nondiabetic and diabetic dams.

Similar observations were also reported in the small litter rat model in which smaller young offspring were exposed to HFD feeding $(4,28,29)$. The implications of those findings were that HFD enhanced insulin sensitivity and fatty-acid oxidation in the skeletal muscle and increased plasma levels of leptin, insulin, and adiponectin to maintain BW and metabolic homeostasis in the normal range $(4,28,29)$. Since increasing lipid oxidation might improve insulin sensitivity $(30,31)$, in this study, when compared with Con-HF mice, Dia-HF animals with normal lipid metabolism and plasma insulin levels had a rapid insulin response and higher insulin sensitivity by IST measurement, hinting that enhanced lipid oxidation might have occurred in these animals. Taken together, our data suggest that Dia-HF animals might use HF as the fuel or energy to balance their BW and metabolism.

In humans, increased intrarenal and perirenal fat is associated with an increased risk of hypertension and chronic kidney disease $(17,18)$. However, the observations of obesity-induced hypertension in animals are varied (32). For instance, Kennedy et al. (32) recently reviewed blood pressure responses in obese mouse models such as Lep ${ }^{\mathrm{ob} / \mathrm{ob}}$, LepR ${ }^{\mathrm{db} / \mathrm{db}}$, and HFD-induced obesity; responses of blood pressure include an increase, decrease, or no change, largely depending on strain, sex, age, environment, and the method of measuring BP. In this study, we did not observe an impact from HFD on the development of hypertension in either Con-HF or Dia-HF mice; our results are similar to reported observations in 5 out of 6 nephrectomized rats fed with HFD (33) and obese ZSF1 rats (34). Previously, the mild hypertension we noted in Dia-ND animals $(9,10)$ was mainly because of renal hypertrophy related to renal hyperfiltration with increased GFR. However, we noted that Dia-HF mice at $20 \mathrm{wk}$ of age showed kidney weight/TL and GFR/TL ratios comparable to Con-ND animals, which might be a partial explanation for their normotensive status.

Renal injury has been reported previously in our Dia-ND mice (9-13). In this work, we found that HFD augmented TGF $\beta 1$ and collagen IV gene expression, increased renal lipid accumulation, as well as heightened oxidative stress in both glomerular and tubular compartments in the kidneys of Dia-HF mice, resulting in significant glomerulosclerosis, tubular fibrosis, and apoptosis. Although our findings are consonant with other reports (14-16), results reported in remnant kidney rats receiving HFD (33) seem to ameliorate these parameters/pathways to prevent kidney injury. Currently, we do not have further explanation for this discrepancy beside species differences. 


\section{Diet and maternal diabetes programming}

\section{Articles}

a

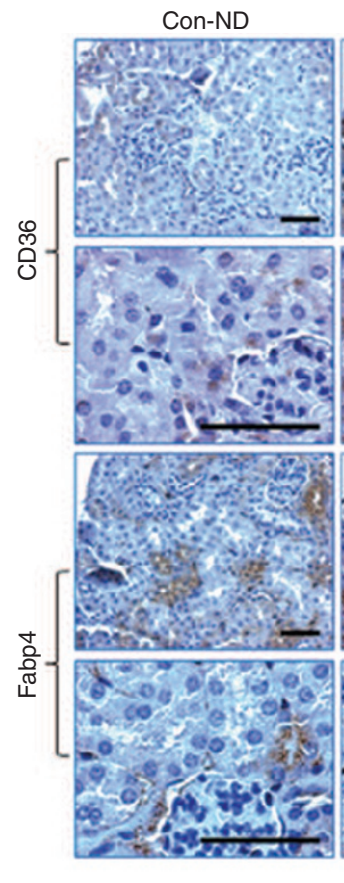

b

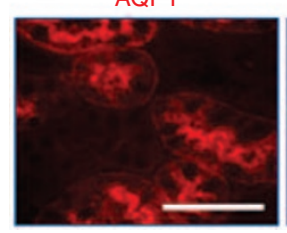

CD36

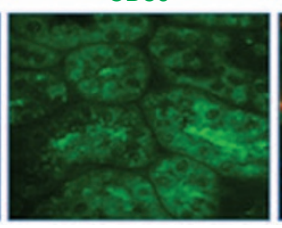

Dia-ND

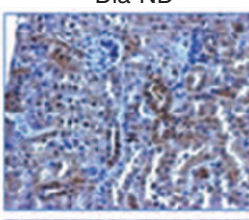

Con-HF

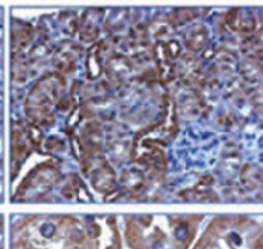

Dia-HF

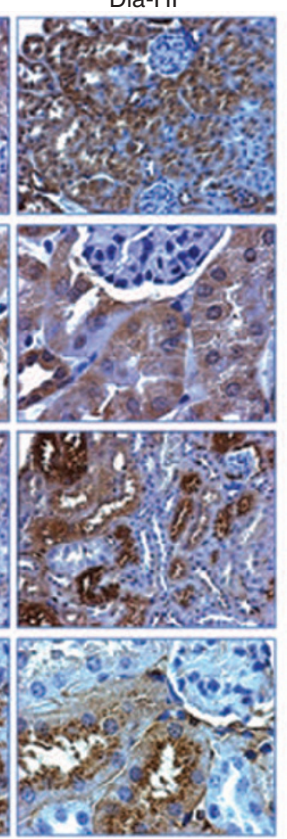

C

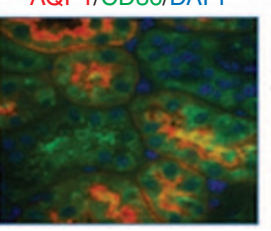

AQP1

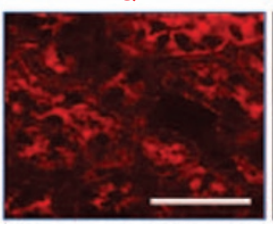

Fabp4

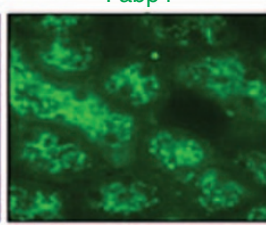

AQP1/Fabp4/DAPI

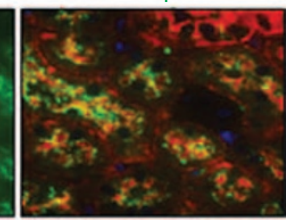

Figure 5. Renal CD36 \& Fabp 4 localization. (a) IHC staining images of CD36 and Fabp4 in the kidneys of four subgroups of 20 wk-old male offspring (magnification: $\times 200 ; \times 600$ ). (b) The colocalization of AQP1 (red) with CD36 (green) in the kidneys of Dia-HF animals (magnification: $\times 600)$. (c) The colocalization of AQP1 (red) with Fabp4 (green) in the kidneys of Dia-HF animals (magnification: $\times 600$ ). AQP1, aquaporin 1; Fabp4, fatty acid-binding protein 4.

Emerging evidence suggested that CD36 (19-22) and Fabp4 (23-27) are highly implicated in the pathogenesis of HFDinduced cell damage. For example, intrarenal CD36 has been identified as a novel mediator in renal diseases associated with proteinuria and renal dysfunction (35). The elevated level of serum/plasma/urinary Fabp4 is considered a predictor or biomarker for renal dysfunction in diabetes and cardiovascular diseases (23-27). Hence, we further hypothesized that these affected IUGR offspring fed with HFD would develop metabolic perturbations and kidney injury in adulthood that might be associated with alterations of both renal CD36 and Fabp4 gene expression.

Our data showed that HFD enhanced renal CD36 and Fabp 4 expression, which predominantly localized to PTCs (confirmed by colocalization in PTCs with the PTC biomarker, aquaporin 1), but not in glomeruli, suggesting that it is plausible that apparent CD36- and Fabp4-mediated PTC changes might be secondary to proteinuria and/or lipiduria.

We validated our in vivo findings with in vitro studies in IRPTCs and investigated the functional impact of free fatty acids (i.e., BSA-PA) on renal CD36 and Fabp 4 expression and its related molecular mechanism(s). We observed that BSA-PA stimulated both CD36 and Fabp4 protein expression in a dose-dependent manner in IRPTCs. CD36 has been shown to mediate PTC apoptosis (36) and to influence the binding and uptake of albumin in PTCs to promote proteinuria (35). The activated CD36 in PTCs targets JNK, ROS, and inflammatory cytokines to facilitate renal dysfunction (22). CD36 deficiency attenuates TGF $\beta 1$ signaling, NF- $\kappa B$ activity, and renal fibrosis in hypercholesterolemic mice (37), whereas CD36 overexpression leads to lipid accumulation in PTCs (20). On the other hand, Fabp4 was reported to be actively expressed in the peritubular and glomerular region to mediate renal dysfunction in diseased kidney (26,27). Fabp4, via the ER stress-JNK pathway, mediated vascular inflammation in the pathogenesis of cardiovascular disorders associated with obesity and diabetes (38). Thus, we tested those potential mechanisms in IRPTCs in culture, and our data suggested that BSA-PA induced ROS generation, enhanced apoptosis (via phosphorylation of p53), and activated NF-kB (i.e., p50/p65) and TGF $\beta 1$ protein expression in IRPTCs. These data would support the concept that CD36 and Fabp 4, induced by free fatty acids, may contribute to the substantial PTC damages resulting in tubulointerstitial fibrosis and apoptosis.

Taken together, our observations suggest a synergistic contribution by $\mathrm{CD} 36$ and Fabp4 to renal dysfunction and injury. Our data indicate that, in addition to HFD-induced glomerular injury, HFD via increased CD36 and Fabp4 expression 


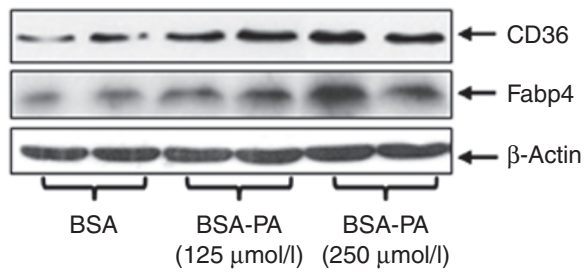

d

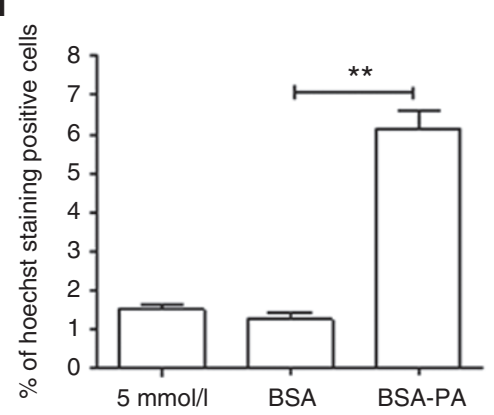

e b

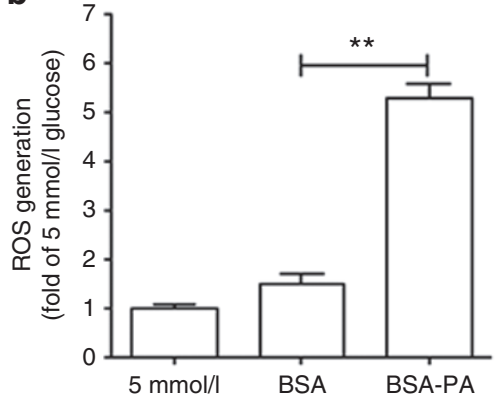

C

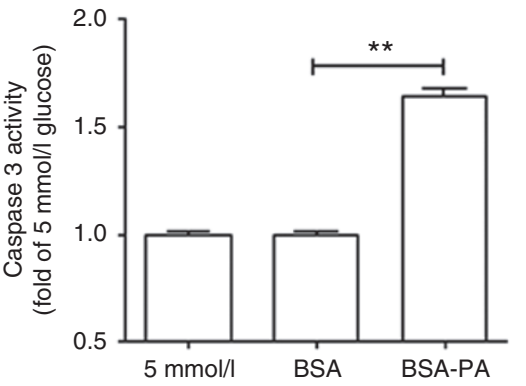

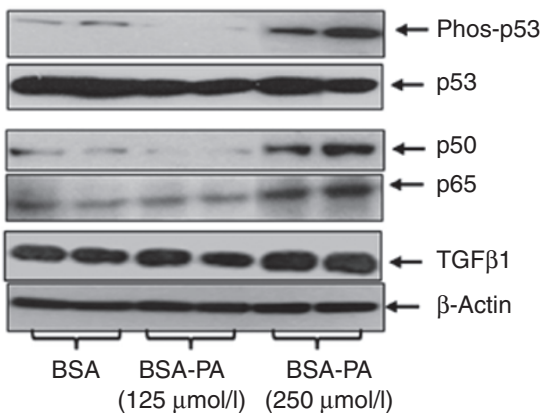

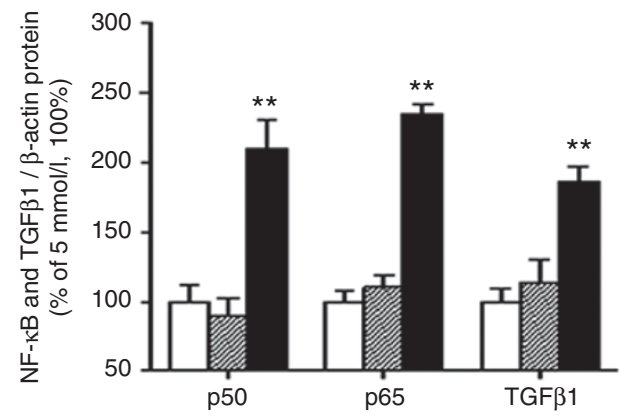

Figure 6. BSA-PA effects in IRPTCs. (a) CD36 and Fabp4 protein expression analyzed by western blot. (b) ROS generation. (c) Caspase 3 activity assay. (d) Quantification of Hoechst staining-positive cells. (e) Western blot of phosphorylation of p53, NF- KB (i.e., p50/p65), and TGF $\beta 1$ protein expression. The relative densities of gene expression were compared with their own $\beta$-actin, and the gene expression protein ratio in $5 \mathrm{mmol} / \mathrm{l}$ glucose medium was considered as $100 \%$. IRPTC in $5 \mathrm{mmol} / \mathrm{I}$ glucose (white bar); BSA (shadowed bar), and BSA-PA (black bar). Each point represents the mean \pm SEM of three independent experiments. ${ }^{*} P \leq 0.05 ;{ }^{* *} P \leq 0.01$. BSA-PA, bovine serum albumin-bound sodium palmitate; IRPTC, immortalized rat renal proximal tubular cell; NF- $\kappa \mathrm{B}$, nuclear factor-kappa B.

in PTCs may target ROS, NF- $\kappa \mathrm{B}$, and TGF $\beta 1$ signaling to accelerate the process of maternal diabetes-induced perinatal programming of kidney injury. In conclusion, early postnatal exposure to HFD in IUGR offspring of diabetic dams increases the risk of development of subsequent kidney injury, but not hypertension.

\section{METHODS}

\section{Animal Models}

In our established in vivo murine model of maternal diabetes induced by a single i.p. streptozotocin $(150 \mathrm{mg} / \mathrm{kg})$ injection on gestational day E13 (9-13), male offspring of diabetic dams displayed more pronounced programming phenotypes; hence, we chose to focus on males for this designed HFD study. Male offspring from nondiabetic and diabetic C57/BL6 mice were fed with normal chow (ND) $(18 \%$ protein with $6.2 \%$ fat, calories from protein $24 \%$, fat $18 \%$, and carbohydrate 58\%; Harlan Teklad, Montreal, Canada) or HFD (20.5\% protein with $36 \%$ fat, calories from protein $14 \%$, fat $60 \%$, and carbohydrate 26\%; Bio-Serv, Flemington, NJ) from 4 wk until 20 wk of age. All animals were killed at $20 \mathrm{wk}$ of age with high $\mathrm{CO}_{2}$, and the kidneys removed immediately. BW (g), kidney weight (mg), and TL $(\mathrm{mm})$ were rapidly recorded. The biological samples were processed, collected, and stored accordingly for analysis. Kidneys were either quickly frozen in OCT or fixed overnight in $4 \%$ paraformaldehyde at $4{ }^{\circ} \mathrm{C}$ before paraffin embedding.

Animal care and the procedures utilized were approved by the Institutional Animal Care Committee of the CRCHUM. Mice were housed under standard humidity and lighting conditions (12-h lightdark cycles) with free access to water and food designed.

\section{Biochemical and Physiological Studies}

Plasma NEFA level ( $\mathrm{mmol} / \mathrm{l})$ was measured using an NEFA kit that detects a variety of free fatty acids (Wako Chemical, Osaka, Japan). Plasma triglycerides $(\mathrm{mg} / \mathrm{ml})$ were determined by GPO Trinder kit (Sigma Aldrich, St Louis, MO). Plasma cholesterol $(\mu \mathrm{g} / \mathrm{ml})$ was assessed by the amplex red cholesterol assay kit (Invitrogen, Burlington, Canada). Plasma insulin was measured by mouse ultrasensitive insulin ELISA jumbo kit (Alpco Diagnostics, Salem, NH).

Blood glucose levels were measured with an Accu-Chek Performa glucose meter (Roche Diagnostics, Laval, Canada) in the morning after a 4 -h fast, as previously reported $(9,10)$. Mean SBP was monitored by the tail-cuff method with the Visitech BP-2000 Blood Pressure Analysis System for mice (Visitech System, Apex, NC) in longitudinal fashion to minimize stress-related hypertension $(9,10)$. The animals were acclimated to SBP measurement with a $2 \mathrm{wk}$ period of pretraining starting at $4 \mathrm{wk}$ of age (SBP measured 15 times/animal/ day, thrice weekly), followed by actual measurement of SBP thrice weekly from $6 \mathrm{wk}$ until $20 \mathrm{wk}$ of age.

\section{Intraperitoneal Glucose Tolerance Test and IST}

Intraperitoneal glucose tolerance test and IST were performed according to a standard protocol. Blood glucose was quantified with an Accu-Chek Performa glucose meter. Briefly, intraperitoneal glucose tolerance test was performed after a 6 - $\mathrm{h}$ fast; $1 \mathrm{mg} / \mathrm{g}$ body glucose was injected intraperitoneally, and blood glucose levels were measured at $0,15,30,60$, and $120 \mathrm{~min}$. IST was initiated after a 4 -h fast. Humulin R (Eli Lilly Canada, Toronto, Canada; 0.75 units $/ \mathrm{kg}$ ) was injected intraperitoneally, and blood glucose levels were measured at $0,15,30,60$, and $90 \mathrm{~min}$.

\section{Glomerular Filtration Rate}

As reported previously (9), we measured GFR in 20 wk-old male animals by the fluorescein isothiocyanate-inulin method, recommended by the Diabetic Complications Consortium. Urine samples, collected from mice individually housed in metabolic cages, were assayed for albumin/creatinine ratio (ELISA, Albuwell and Creatinine Companion, Exocell, Philadelphia, PA), as reported previously $(9,10)$.

\section{Histology}

We assessed hematoxylin and eosin staining of adipocyte collected from both visceral and perirenal fat. The number of adipocytes 
displayed in microscopic fields ( $N=10$ fields per animal) were quantitated in a randomized and blinded fashion. Renal morphology was assessed with periodic acid-Schiff and Masson's trichrome staining $(9,10)$. IHC was performed by the standard avidin-biotin-peroxidase complex method (Santa Cruz Biotechnologies, Santa Cruz, CA), as described elsewhere $(9,10)$. The antibodies used included polyclonal p53 and aquaporin 1 (Santa Cruz Biotechnologies); anti-TGF $\beta 1$ antibody (R\&D Systems, Burlington, Canada); monoclonal anticollagen type IV antibody (Chemicon International, Temecula, CA); polyclonal CD36 antibody (Abcam, Cambridge, MA); Fabp4 antibody (R\&D Systems). Oxidative stress in vivo was assessed by dihydroethidium (Sigma-Aldrich, Oakville, Canada) staining in frozen kidney sections as reported previously (13). The classic scoring of glomerulosclerosis (scale from 0 to 4 ) (39) and tubulo-interstitial injury (scale from 0 to 3) (40) was based on periodic acid-Schiff images. The semi-quantitation of the relative staining values was performed by NIH Image J software (Bethesda, MD) $(9,10)$. The images $(N=$ 6-8 per animal) were analyzed and quantitated in a randomized and blinded fashion.

\section{Real-Time Quantitative PCR}

Total RNA extracted from freshly isolated renal cortex was assayed for gene expression by real-time quantitative PCR and calculated using the $\Delta \Delta C_{\mathrm{t}}$ method, as reported previously (9-13). The Fast SYBR green mastermix kit and the 7500 Fast real-time PCR system (Applied Biosystems, Life Technologies, Foster City, CA) were employed for this purpose (9-13). The primer sequences are listed as: CD36 (S), $5^{\prime}$-catattggtcaagccagctag- $3^{\prime}$; CD36 (AS), $5^{\prime}$-agcaacaaacatcaccactcc-3'; Fabp4 (S), 5'-aaggtgaagagcatcataaccct-3'; Fabp4 (AS), $5^{\prime}$-tcacgcctttcataacacattcc- $3^{\prime}$.

\section{In Vitro Studies}

The IRPTC line (passages 15-18) reported previously (9) was employed for our in vitro studies. Caspase- 3 activity, ROS generation, and Hoechst staining were measured in IRPTCs treated with or without BSA (fatty acid free; Sigma-Aldrich) or (BSA-PA (Sigma-Aldrich) overnight. The preparation of BSA-PA $(125 \mu \mathrm{mol} / \mathrm{l}$ and $250 \mu \mathrm{mol} / \mathrm{l})$ was described in detail by Roduit et al (41). The antibodies used for western blot included NF-kB (p50/p65), p53, and TGF 31 from Santa Cruz Biotechnology; anti-phospho-p53 (Ser 15) from Abcam.

\section{Statistical Analysis}

Statistical significance between the experimental groups was analyzed by one-way ANOVA, by using Graphpad Software, Prism 5.0 (La Jolla, CA). A probability level of $P \leq 0.05$ was considered to be statistically significant and followed by a Bonferroni analysis with adjustment for multiple comparisons (9-13).

\section{ACKNOWLEDGMENTS}

The authors owe special thanks to John S.D. Chan (CRCHUM, Montreal, QC, Canada) for his unconditional support and valuable comments on this manuscript. Editorial assistance was provided by the CRCHUM's Research Support Office.

\section{STATEMENT OF FINANCIAL SUPPORT}

This project was supported by grants to S.-L.Z. from the Canadian Institutes of Health Research (MOP115025) and Canadian Diabetes Association (OG3-13-4073-SZ).

\section{Disclosure: None.}

\section{REFERENCES}

1. Plagemann A. Maternal diabetes and perinatal programming. Early Hum Dev 2011;87:743-7.

2. Plagemann A, Harder T, Rodekamp E, Kohlhoff R. Rapid neonatal weight gain increases risk of childhood overweight in offspring of diabetic mothers. J Perinat Med 2012;40:557-63.

3. Plagemann A, Harder T, Schellong K, Schulz S, Stupin JH. Early postnatal life as a critical time window for determination of long-term metabolic health. Best Pract Res Clin Endocrinol Metab 2012;26:641-53.

4. Spencer SJ. Early life programming of obesity: the impact of the perinatal environment on the development of obesity and metabolic dysfunction in the offspring. Curr Diabetes Rev. 2012;8:55-68.
5. Holemans K, Aerts L, Van Assche FA. Fetal growth restriction and consequences for the offspring in animal models. J Soc Gynecol Investig 2003;10:392-9.

6. Van Assche FA, Holemans K, Aerts L. Long-term consequences for offspring of diabetes during pregnancy. Br Med Bull 2001;60:173-82.

7. Perälä MM, Männistö S, Kaartinen NE, et al. Body size at birth is associated with food and nutrient intake in adulthood. PLoS One 2012;7:e46139.

8. Crowther NJ, Cameron N, Trusler J, Toman M, Norris SA, Gray IP. Influence of catch-up growth on glucose tolerance and beta-cell function in 7-year-old children: results from the birth to twenty study. Pediatrics 2008;121:e1715-22.

9. Chang SY, Chen YW, Zhao XP, et al. Catalase prevents maternal diabetesinduced perinatal programming via the Nrf2-HO-1 defense system. Diabetes 2012;61:2565-74.

10. Chen YW, Chenier I, Tran S, Scotcher M, Chang SY, Zhang SL. Maternal diabetes programs hypertension and kidney injury in offspring. Pediatr Nephrol 2010;25:1319-29.

11. Tran S, Chen YW, Chenier I, et al. Maternal diabetes modulates renal morphogenesis in offspring. J Am Soc Nephrol 2008;19:943-52.

12. Zhang SL, Chen YW, Tran S, Chenier I, Hébert MJ, Ingelfinger JR. Reactive oxygen species in the presence of high glucose alter ureteric bud morphogenesis. J Am Soc Nephrol 2007;18:2105-15.

13. Chen YW, Chenier I, Chang SY, Tran S, Ingelfinger JR, Zhang SL. High glucose promotes nascent nephron apoptosis via NF-kappaB and p53 pathways. Am J Physiol Renal Physiol 2011;300:F147-56.

14. Declèves AE, Mathew AV, Cunard R, Sharma K. AMPK mediates the initiation of kidney disease induced by a high-fat diet. J Am Soc Nephrol 2011;22:1846-55.

15. Odermatt A. The Western-style diet: a major risk factor for impaired kidney function and chronic kidney disease. Am J Physiol Renal Physiol 2011;301:F919-31.

16. Soumura $\mathrm{M}$, Kume $\mathrm{S}$, Isshiki $\mathrm{K}$, et al. Oleate and eicosapentaenoic acid attenuate palmitate-induced inflammation and apoptosis in renal proximal tubular cell. Biochem Biophys Res Commun 2010;402:265-71.

17. Foster MC, Hwang SJ, Porter SA, Massaro JM, Hoffmann U, Fox CS. Fatty kidney, hypertension, and chronic kidney disease: the Framingham Heart Study. Hypertension 2011;58:784-90.

18. Chandra A, Neeland IJ, Berry JD, et al. The relationship of body mass and fat distribution with incident hypertension: observations from the Dallas Heart Study. J Am Coll Cardiol 2014;64:997-1002.

19. Hajri T, Han XX, Bonen A, Abumrad NA. Defective fatty acid uptake modulates insulin responsiveness and metabolic responses to diet in CD36-null mice. J Clin Invest 2002;109:1381-9.

20. Kang HM, Ahn SH, Choi P, et al. Defective fatty acid oxidation in renal tubular epithelial cells has a key role in kidney fibrosis development. Nat Med 2015;21:37-46.

21. Kennedy DJ, Kuchibhotla S, Westfall KM, Silverstein RL, Morton RE, Febbraio M. A CD36-dependent pathway enhances macrophage and adipose tissue inflammation and impairs insulin signalling. Cardiovasc Res 2011;89:604-13.

22. Kennedy DJ, Chen Y, Huang W, et al. CD36 and Na/K-ATPase- $\alpha 1$ form a proinflammatory signaling loop in kidney. Hypertension 2013;61:216-24.

23. Ishimura S, Furuhashi M, Watanabe $Y$, et al. Circulating levels of fatty acidbinding protein family and metabolic phenotype in the general population. PLoS One 2013;8:e81318.

24. Furuhashi M, Ishimura $\mathrm{S}$, Ota $\mathrm{H}$, et al. Serum fatty acid-binding protein 4 is a predictor of cardiovascular events in end-stage renal disease. PLoS One 2011;6:e27356.

25. Yeung DC, Xu A, Tso AW, et al. Circulating levels of adipocyte and epidermal fatty acid-binding proteins in relation to nephropathy staging and macrovascular complications in type 2 diabetic patients. Diabetes Care 2009;32:132-4.

26. Tanaka M, Furuhashi M, Okazaki Y, et al. Ectopic expression of fatty acidbinding protein 4 in the glomerulus is associated with proteinuria and renal dysfunction. Nephron Clin Pract 2014;128:345-51.

27. Okazaki Y, Furuhashi M, Tanaka $M$, et al. Urinary excretion of fatty acidbinding protein 4 is associated with albuminuria and renal dysfunction. PLoS One 2014;9:e115429. 


\section{Articles | Aliou e tal.}

28. Prior LJ, Velkoska E, Watts R, Cameron-Smith D, Morris MJ. Undernutrition during suckling in rats elevates plasma adiponectin and its receptor in skeletal muscle regardless of diet composition: a protective effect? Int J Obes (Lond) 2008;32:1585-94.

29. Velkoska E, Cole TJ, Dean RG, Burrell LM, Morris MJ. Early undernutrition leads to long-lasting reductions in body weight and adiposity whereas increased intake increases cardiac fibrosis in male rats. J Nutr 2008;138:1622-7.

30. Bruce CR, Hoy AJ, Turner N, et al. Overexpression of carnitine palmitoyltransferase-1 in skeletal muscle is sufficient to enhance fatty acid oxidation and improve high-fat diet-induced insulin resistance. Diabetes 2009;58:550-8.

31. Murrow BA, Hoehn KL. Mitochondrial regulation of insulin action. Int J Biochem Cell Biol 2010;42:1936-9.

32. Kennedy AJ, Ellacott KL, King VL, Hasty AH. Mouse models of the metabolic syndrome. Dis Model Mech 2010;3:156-66.

33. Kim HJ, Vaziri ND, Norris K, An WS, Quiroz Y, Rodriguez-Iturbe B. High-calorie diet with moderate protein restriction prevents cachexia and ameliorates oxidative stress, inflammation and proteinuria in experimental chronic kidney disease. Clin Exp Nephrol 2010;14:536-47.

34. Hamdani N, Franssen C, Lourenço A, et al. Myocardial titin hypophosphorylation importantly contributes to heart failure with preserved ejection fraction in a rat metabolic risk model. Circ Heart Fail 2013;6:1239-49.
35. Baines RJ, Chana RS, Hall M, Febbraio M, Kennedy D, Brunskill NJ. CD36 mediates proximal tubular binding and uptake of albumin and is upregulated in proteinuric nephropathies. Am J Physiol Renal Physiol 2012;303:F1006-14.

36. Susztak K, Ciccone E, McCue P, Sharma K, Böttinger EP. Multiple metabolic hits converge on CD36 as novel mediator of tubular epithelial apoptosis in diabetic nephropathy. PLoS Med 2005;2:e45.

37. Okamura DM, López-Guisa JM, Koelsch K, Collins S, Eddy AA. Atherogenic scavenger receptor modulation in the tubulointerstitium in response to chronic renal injury. Am J Physiol Renal Physiol 2007;293:F575-85.

38. Xu A, Vanhoutte PM. Adiponectin and adipocyte fatty acid binding protein in the pathogenesis of cardiovascular disease. Am J Physiol Heart Circ Physiol 2012;302:H1231-40.

39. el Nahas AM, Bassett AH, Cope GH, Le Carpentier JE. Role of growth hormone in the development of experimental renal scarring. Kidney Int 1991;40:29-34.

40. Véniant M, Heudes D, Clozel JP, Bruneval P, Ménard J. Calcium blockade versus ACE inhibition in clipped and unclipped kidneys of $2 \mathrm{~K}-1 \mathrm{C}$ rats. Kidney Int 1994;46:421-9.

41. Roduit R, Masiello P, Wang SP, Li H, Mitchell GA, Prentki M. A role for hormone-sensitive lipase in glucose-stimulated insulin secretion: a study in hormone-sensitive lipase-deficient mice. Diabetes 2001;50: 1970-5. 